\title{
THE ECOLOGY OF MAGNETIC ROTATORS
}

\author{
V.M. LIPUNOV \\ Sternberg Astronomical Institute \\ Universitetskij pr. 13, 119899, \\ Moscow V-234, Russia
}

\begin{abstract}
A review is given concerning the current state of the theory of evolution of magnetic compact stars. The intrinsic evolution of the magnetized compact star is shown, both theoretically and numerically, to be the decisive factor in explaining observable properties, and in predicting yet unknown properties of high-energy radiation sources in our and other galaxies. The main results are given of recent evolutionary scenario simulations (Scenario Machine) by the Monte-Carlo method.
\end{abstract}

\section{Introduction}

It became clear in the early 1980's that the level of comprehension of stellar evolution theory on the one hand, and the observed variety of astrophysical properties of stars gained in numerous space experiments on the other hand, approached a point where construction of particular scenarios for newly discovered exotic sources could be a viable approach. The importance of involving not only a general evolutionary scenario for the joint evolution of normal and compact stars, but also numerical simulations, using the so-called Scenario Machine (Lipunov 1991) became quite obvious (Lipunov 1982a). The first numerical simulation of the evolution of massive binary systems taking into account rotation of neutron stars was carried out by Kornilov \& Lipunov (1983a,b; 1984). Subsequently, the ideas and method were extrapolated to low-mass stars (Lipunov \& Postnov 1987, 1988). Finally, we now have a general scenario and numerical model for the evolution of binary systems with arbitrary masses (see, e.g., Tatarintzeva et al. 1989; Lipunov et al. 1994a,b). 
TABLE 1. Classification of Magnetic Rotators

\begin{tabular}{|c|c|c|c|c|}
\hline & Type & $\begin{array}{l}\text { Relation between } \\
\text { characteristic radii }\end{array}$ & $\begin{array}{l}\text { Accretion } \\
\text { rate }\end{array}$ & $\begin{array}{l}\text { Observational } \\
\text { appearances }\end{array}$ \\
\hline $\mathrm{E}$ & ejector & $\begin{array}{l}R_{\mathrm{st}}>R_{\mathrm{G}} \\
R_{\mathrm{st}}>R_{\mathrm{l}}\end{array}$ & $\dot{M}_{\mathrm{c}} \leq \dot{M}_{\mathrm{cr}}$ & $\begin{array}{l}\text { radio pulsars } \\
\text { soft } \gamma \text {-ray repeater } \\
\text { Cyg X-3? LS I }+61^{\circ} 303 ?\end{array}$ \\
\hline $\mathbf{P}$ & propeller & $\begin{array}{l}R_{\mathrm{c}}<R_{\mathrm{st}} \\
R_{\mathrm{st}} \leq \max \left\{R_{\mathrm{G}}, R_{\mathrm{l}}\right\}\end{array}$ & $\begin{array}{l}\dot{M}_{\mathrm{c}} \leq \dot{M}_{\mathrm{cr}} \\
\text { rapid burster? }\end{array}$ & $\begin{array}{l}\text { X-ray transients? } \\
\gamma \text {-bursters??? } \\
\text { magnetic Ap stars }\end{array}$ \\
\hline A & accretor & $\begin{array}{l}R_{\mathrm{st}} \leq R_{\mathrm{G}} \\
R_{\mathrm{st}} \leq R_{\mathrm{l}}\end{array}$ & $\dot{M}_{\mathrm{c}} \leq \dot{M}_{\mathrm{cr}}$ & $\begin{array}{l}\text { X-ray pulsars, } \\
\text { bursters, CVs } \\
\text { intermediate polars }\end{array}$ \\
\hline G & georotator & $\begin{array}{l}\boldsymbol{R}_{\mathrm{G}}<\boldsymbol{R}_{\mathrm{st}} \\
\boldsymbol{R}_{\mathrm{st}} \leq \boldsymbol{R}_{\mathrm{c}}\end{array}$ & $\dot{M}_{\mathrm{c}} \leq \dot{M}_{\mathrm{cr}}$ & Earth, Jupiter \\
\hline $\mathbf{M}$ & magnetor & $\begin{array}{l}R_{\mathrm{st}}>a \\
R_{\mathrm{c}}>a ? ? ?\end{array}$ & $\dot{M}_{\mathrm{c}} \leq \dot{M}_{\mathrm{cr}}$ & AM Her, polars \\
\hline $\mathrm{SE}$ & superejector & $R_{\mathrm{st}}>R_{\mathrm{l}}$ & $\dot{M}_{\mathrm{c}}>\dot{M}_{\mathrm{cr}}$ & $?$ \\
\hline SP & superpropeller & $\begin{array}{l}R_{\mathrm{c}}<R_{\mathrm{st}} \leq R_{\mathrm{l}} \\
R_{\mathrm{st}} \leq R_{\mathrm{l}}\end{array}$ & $\dot{M}_{\mathrm{c}}>\dot{M}_{\mathrm{cr}}$ & $?$ \\
\hline $\mathrm{SA}$ & superaccretor & $\begin{array}{l}R_{\mathrm{st}} \leq R_{\mathrm{c}} \\
R_{\mathrm{st}} \leq R_{\mathrm{G}}\end{array}$ & $\dot{M}_{\mathrm{c}}>\dot{M}_{\mathrm{cr}}$ & $\begin{array}{l}\text { SS } 433 ? \\
\text { T Tau stars? } \\
\text { ultrasoft superluminous } \\
\text { sources? }\end{array}$ \\
\hline
\end{tabular}

\section{Classification of Magnetic Rotators and Binary Systems.}

The current scenario for the evolution of binaries based upon the original ideas which first appeared in Paczyński (1971), Tutukov \& Yungelson (1973), Van den Heuvel \& Heise (1972), was joined with the ideas of neutron star evolution [see the pioneering works by Shvartsman $(1970,1971)$, as well as Mlarionov \& Sunyaev (1975), Bisnovatyi-Kogan \& Komberg (1975), Shakura (1975), Wickramasinghe \& Whelan (1975), Lipunov \& Shakura (1976), Savonije \& Van den Heuvel (1977), and Lipunov (1982a)]. This joint scenario has allowed the construction of a two-dimensional classification of all possible states of binary systems containing neutron stars (Kornilov \& Lipunov 1983a).

The full classification of neutron stars and white dwarfs (magnetic rotators) is based upon the idea that the astrophysical manifestations of these stars mainly reflect the character of the interaction of their electromag- 
netic fields with the surrounding plasma which tends to accrete under the influence of gravitational field.

The complete classification of magnetic compact stars includes eight types (Lipunov 1982a, 1984; see also Lipunov 1992).

\subsection{NOMENCLATURE}

The interaction of a magnetic rotator with the surrounding plasma to a large extent depends on the relative sizes of four characteristic radii: the stopping radius, $R_{\mathrm{st}}$, the gravitational capture radius, $R_{\mathrm{G}}$, the light cylinder radius, $R_{1}$, and the corotation radius, $R_{\mathrm{c}}$. The differences between the interaction regimes are so significant, that the magnetic rotators show entirely different behaviour in different regimes. Hence the classification of the interaction regimes may well mean the classification of magnetic rotators. The classification notation and terminology are described below, and summarized in Table 1 (based on Lipunov 1987).

Naturally, not all possible combinations of the characteristic radii can be realized. For example, the inequality $R_{1}>R_{\mathrm{c}}$ is not possible in principle. Furthermore, some combinations require unrealistically large or small parameters of magnetic rotators. For the same proper and external conditions the same rotator may gradually pass through several interaction regimes. Such a process will be termed the evolution of a magnetic rotator.

\subsection{CLASSIFICATION OF NORMAL STARS IN BINARY SYSTEMS}

The four basic evolutionary stages characterizing a normal star in a binary system can be specified as follows:

I - The size of the star is much smaller than the critical Roche lobe (usually such a star lies on the main sequence). The duration of the first stage is approximately equal to the hydrogen burning time $\left(T_{\mathrm{H}}\right)$;

II - The star leaves the main sequence and goes up to the supergiant region (as before, the star does not fill the Roche lobe). The duration of this stage is about one tenth of $T_{\mathrm{H}}$;

III - The star fills the Roche lobe and begins to flow out to the companion. For large mass ratios, the flow takes place on the thermal time scale of the mass donor;

IV - In the process of mass exchange the envelope of the star flows over and a helium star forms (WR-star). The lifetime of the helium star is determined by the nuclear burning of helium.

In Table 2 we present the two-dimensional classification of massive binary systems with neutron stars and show a matrix of observational candidates. 
TABLE 2. Massive Binaries with Neutron Stars; the Matrix of States.

\begin{tabular}{lccccc}
\hline & $\begin{array}{c}\text { I } \\
\text { main } \\
\text { sequence }\end{array}$ & $\begin{array}{c}\text { II } \\
\text { supergiant }\end{array}$ & $\begin{array}{c}\text { III } \\
\text { Roche lobe } \\
\text { overflow }\end{array}$ & $\begin{array}{c}\text { IV } \\
\text { Wolf-Rayet } \\
\text { star }\end{array}$ & NS \\
\hline E & PSR $1259-63^{1}$ & $?$ & $?$ & Cyg X-3(?) & PSR 1913+16 \\
P & $?$ & $?$ & $?$ & $?$ & $?$ \\
A & A 0535+26 & Cen X-3 & Her X-1 & $?$ & $?$ \\
G & $?$ & $?$ & - & $?$ & $?$ \\
M & $?$ & - & - & - & $?$ \\
SE & $?$ & $?$ & $?$ & $?$ & $?$ \\
SP & $?$ & $?$ & $?$ & $?$ & $?$ \\
SA & - & - & SS 433(?) & - & $?$ \\
BH & $?$ & Cyg X-1 & $?$ & $?$ & PSR B0042-73(?) \\
SBH & - & - & SS 433(?) & $?$ & - \\
\hline
\end{tabular}

1) Johnston, S. et al. 1992

2) Van Kerkwijk, M.H. et al. 1992

3) Kaspi, V.M. et al. 1994

4) Lipunov, V.M., Postnov, K.A. \& Prokhorov, M.E. 1994c

\section{Evolution of Neutron Stars}

The evolution of neutron stars must be investigated taking into account the evolution of normal stars. This problem was discussed qualitatively by Bisnovatyi-Kogan \& Komberg (1974), Van den Heuvel (1977) and Lipunov (1982a). We begin with the qualitative analysis presented in the latter paper.

The most convenient way of analyzing the evolution of a neutron star is with the help of the $P-y$ diagram (Lipunov 1987). In the expression for the stopping radius in the subcritical regime $\left(\dot{M}_{\mathrm{c}} \geq \dot{M}_{\mathrm{cr}}\right)$ one notes that the magnetic dipole moment $\mu$ and the accretion rate $\dot{M}_{\mathrm{c}}$ always appear in the same combination,

$$
y=\frac{\dot{M}_{\mathrm{c}}}{\mu^{2}},
$$

as was first noticed by Davies \& Pringle (1981). The parameter $y$ characterizes the ratio between the gravitational and magnetic "properties" of a star and will, therefore, be called the gravimagnetic parameter. Two magnetic rotators having quite diffe:ent magnetic fields and external conditions but identical gravimagnetic parameters have similar magnetospheres, as long as the accretion rate is quite low $\left(\dot{M}_{\mathrm{c}} \leq \dot{M}_{\mathrm{cr}}\right)$. Otherwise, the flux of matter 


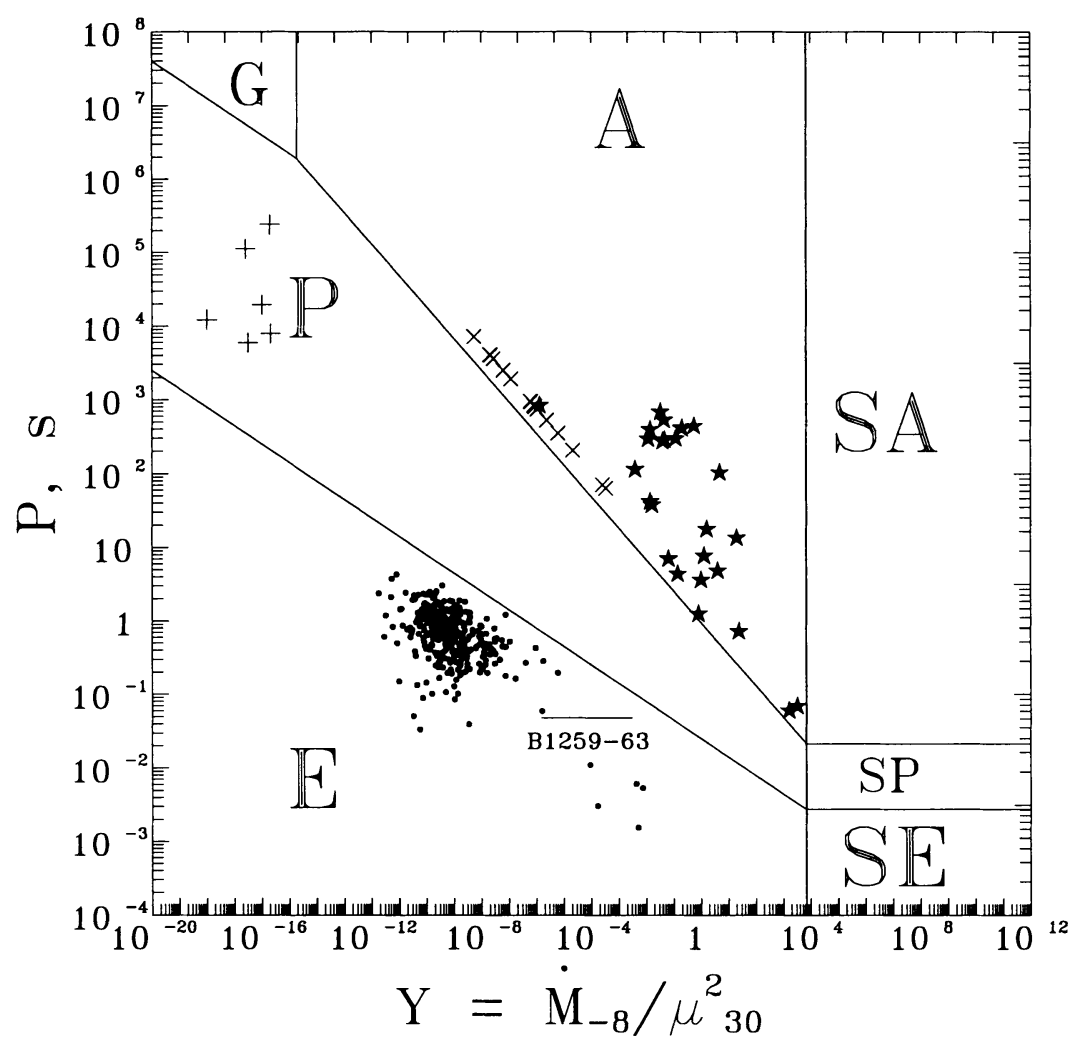

Figure 1. "P $-y$ " diagram for neutron stars and white dwarfs. This version of the diagram contains binary WD (intermediate polars - $\times$ ), isolated WD (cross), isolated NS (radio pulsars - dots), binary accreting NS (X-ray pulsars - asterisks) (Lipunov, Postnov \& Prokhorov 1995).

near the stopping radius no longer depends on the accretion rate at a large distance.

In fact, the number of independent parameters can be further reduced (see Osminkin \& Prokhorov 1994) by introducing the parameter

$$
y=\frac{\dot{M}_{\mathrm{c}} v_{\infty}}{\mu^{2}}
$$

Plotting the rotator's period $P$ versus $y$ we obtain a somewhat less obvious, but more general, classification diagram than the " $P-L$ " diagram discussed in Lipunov (1982a) or the " $P-B$ " diagram (see, e.g., Ghosh, these proceedings).

In Fig. 2 we show the evolutionary tracks of neutron stars. As a rule, the neutron star is generated at the instant when the companion lies on the main sequence (track b). During the first $10^{5-7}$ years, the neutron star 


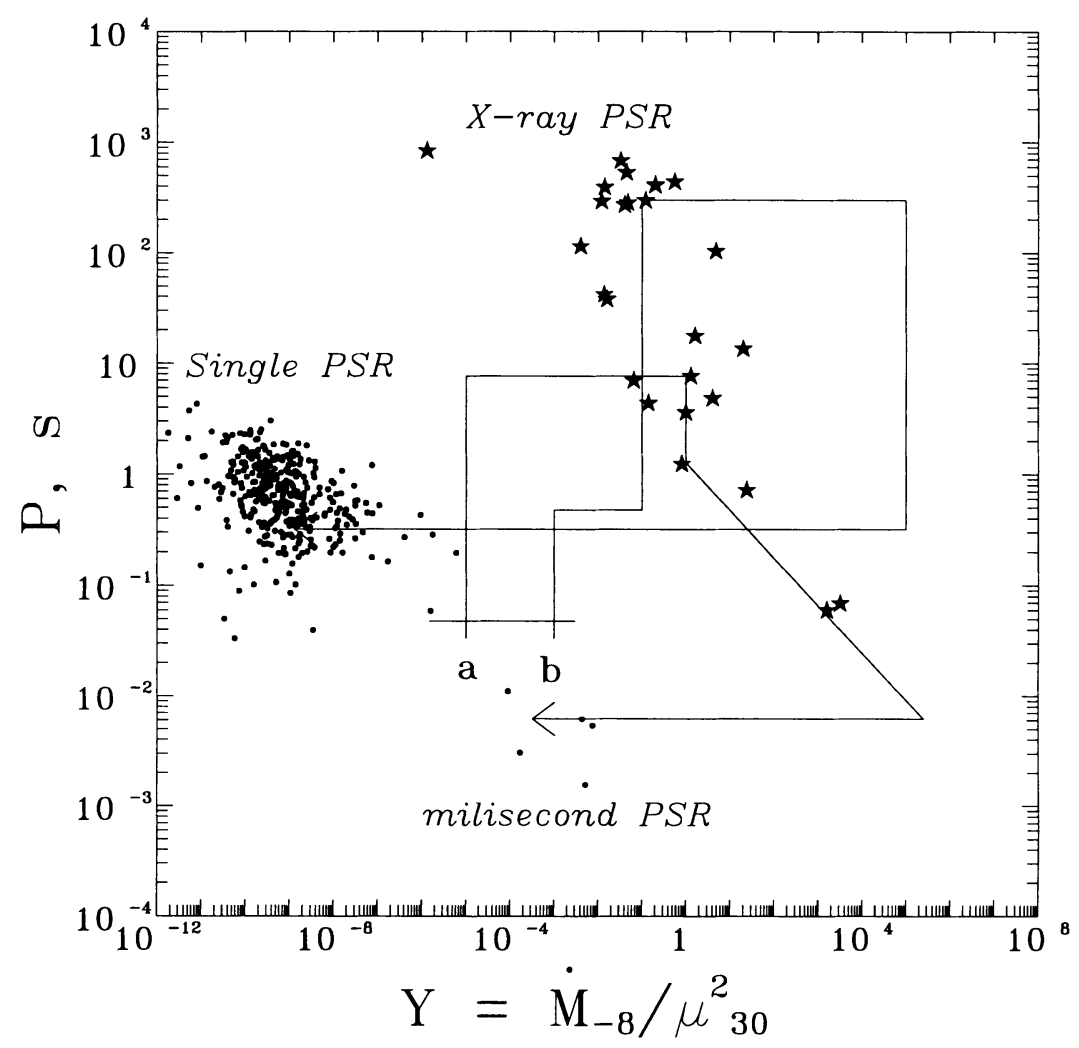

Figure 2. Tracks of neutron stars on a period $(P)$ versus gravimagnetic parameter $(y)$ diagram. (b) - track of a neutron star with standard magnetic field in a massive binary; (a) - track of a neutron star with magnetic-field decay in a binary system with a low-mass companion.

is an ejector; usually it does not manifest itself as a radio pulsar since its radio waves are absorbed in the stellar wind of the normal star. The period of the neutron star increases in accordance with the magnetic dipole losses.

After this, matter penetrates into the light cylinder and the neutron star passes first into the propeller stage, and then into the accretor stage. By this time, the normal star leaves the main sequence and the stellar wind is intensified. This results in the generation of a bright X-ray pulsar. The period of the neutron star stabilizes around its equilibrium value. Finally, the normal star fills its Roche lobe and the accretion rate suddenly increases; the neutron star moves first to the right and then vertically down on the $P-y$ diagram. In other words, the neutron star moves into the supercritical stage SA (superaccretor). Its period tends to a new equilibrium value (see Lipunov 1992):

$$
P_{\mathrm{eq}}(\mathbf{S A}) \simeq(0.17 s) \mu_{30}^{2 / 3} m_{\mathrm{X}}^{-1 / 9}
$$


After the mass exchange, only the helium core of the normal star is left (WR star), a separate system is formed, and the neutron star reverts to the propeller or ejector stage. Accretion is hampered by rapid rotation. This is probably the reason behind the absence of X-ray pulsars in pairs with Wolf-Rayet stars (Lipunov 1982b). Since the helium star does not have a long life $\left(\sim 10^{5} \mathrm{yrs}\right)$, the neutron star does not have time to spin down considerably: after the explosion of the normal star, the system disintegrates and the neutron star becomes an ejector, that is, a radio pulsar.

The "loop-shaped" track discussed above can be written in the form:

$$
\begin{aligned}
\text { I E } \rightarrow \text { I P } \rightarrow \text { II P } \rightarrow & \text { II A } \rightarrow \text { III SA } \rightarrow \\
& \text { IV P } \rightarrow \mathbf{E}+\mathbf{E}(\text { recycled pulsar }) \rightarrow \ldots \\
\text { I E } \rightarrow \text { I P } \rightarrow \text { II A } \rightarrow & \text { III SA } \rightarrow \text { IV E }(\text { recycled ejector }) \rightarrow \\
& \text { IV P } \rightarrow \mathbf{E}+\mathbf{E}(\text { recycled pulsar }) \rightarrow \ldots
\end{aligned}
$$

The overall lifetime of a neutron star in a binary system depends on the lifetime of the normal star and the parameters of the binary system. However, the rate of transition from one stage to another is proportional to the magnetic field strength of the neutron star.

\subsection{EVOLUTION EQUATION}

An analysis of the nature of the interaction of a magnetized star with the surrounding plasma allows us to write the approximate evolutionary equation for the angular momentum of a magnetic rotator in the general form (Lipunov 1982a)

$$
\frac{d I \omega}{d t}=\dot{M} k_{\mathrm{su}}-\kappa_{\mathrm{t}} \frac{\mu^{2}}{R_{\mathrm{t}}^{3}}
$$

where $k_{\text {su }}$ is the specific angular momentum applied by the accretion matter to the rotator. This quantity is given by $k_{\mathrm{su}}=\left(G M_{\mathrm{X}} R_{\mathrm{d}}\right)^{1 / 2}$ for Keplerian disk accretion, by $k_{\mathrm{su}}=\eta_{\mathrm{t}} \Omega R_{\mathrm{G}}^{2}$ for wind accretion in a binary, and $k_{\mathrm{su}} \sim 0$ for a single magnetic rotator. Here $R_{\mathrm{d}}$ is the radius of the inner disk edge, $\Omega$ is the rotational frequency of the binary system, and $\eta_{\mathrm{t}} \sim 1 / 4$ (Illarionov \& Sunyaev 1975). The values of the dimensionless factor $\kappa_{\mathrm{t}}$, the characteristic radius $R_{\mathrm{t}}$ and the accretion rate $\dot{M}$ in different regimes are presented in Table 3.

The evolution equation is approximate. In practice, the situation concerning propellers and superpropellers is not yet clear. In Table $3 R_{\mathrm{m}}$ is the size of the magnetosphere whose value at the propeller stage is not known 
TABLE 3. Parameters of the Evolution Equation of a Magnetic Rotator

\begin{tabular}{lcccccc}
\hline \multirow{2}{*}{ Parameter } & \multicolumn{6}{c}{ Regime } \\
\cline { 2 - 7 } & $\mathrm{E}, \mathrm{SE}$ & $\mathrm{P}, \mathrm{SP}$ & $\mathrm{A}$ & $\mathrm{SA}$ & $\mathrm{G}$ & $\mathrm{M}$ \\
\hline$\dot{M}$ & 0 & 0 & $\dot{M}_{\mathrm{c}}$ & $\dot{M}_{\mathrm{c}}\left(R_{\mathrm{A}} / R_{\mathrm{s}}\right)$ & 0 & $\dot{M}_{\mathrm{c}}$ \\
$\kappa_{\mathrm{t}}$ & $\sim 2 / 3$ & $\lesssim 1 / 3$ & $\sim 1 / 3$ & $\sim 1 / 3$ & $\sim 1 / 3$ & $\sim 1 / 3$ \\
$R_{\mathrm{t}}$ & $R_{\mathrm{l}}$ & $R_{\mathrm{m}}$ & $R_{\mathrm{c}}$ & $R_{\mathrm{c}}$ & $R_{\mathrm{A}}$ & $a$ \\
\hline
\end{tabular}

accurately, and may differ significantly from the standard expressions for the Alfvén radius.

\section{Scenario Machine: Computational Method.}

To analyze the properties of an ensemble of binaries in the Galaxy, a special numerical program has been designed. In essence, it is a rough model of the real Galaxy. Calculations are carried out using the Monte-Carlo method. A binary system consisting of two normal stars is chosen. The time of its formation is drawn at random, and so are its binary parameters, which are distributed in accordance with currently established empirical laws. The binary system evolves in accordance with the pattern described above. Each star gradually passes through the stages I, II, III, IV. The parameters of the system and the outflow of matter from it are assumed to be constant within each stage. The duration of the stages is calculated from approximation formulae (see, e.g., Lipunov \& Postnov 1988). After the appearance of a neutron star or white dwarf in the binary system, the magnetized compact star evolution block is introduced in accordance with the spin evolution equation (see Lipunov 1992). Obviously, the state of the binary system is described by a two-dimensional classification (Kornilov \& Lipunov 1983a). For example, state IIA means that we are dealing with a binary system in which the normal star is in stage II, with a supergiant that does not fill its Roche lobe, while the neutron star is in the accretor stage. A typical representative of such systems is the classical massive binary X-ray pulsar Vela X-1. The method of calculating the joint evolution of a NS and the normal companion in the binary system for statistical comparison with observed characteristics (and also for predicting the hitherto undefined stages of evolution of massive binaries) is based on the calculation of the evolution of large number (about $10^{6}$ systems in one experiment) of binary systems with randomly chosen parameters. 


\section{Monte Carlo Scenario Machine History}

The following five main physical processes must be included into the modern scenario of the binary-star evolution:

a Mass exchange;

b Loss of orbital angular momentum due to gravitational waves;

c Loss of orbital angular momentum due to magnetic wind;

d Evaporation of the primary star by a radio pulsar companion;

e Magnetic compact star spin evolution.

History:

$a+e$; Massive binaries - Kornilov \& Lipunov (1983a,b,1984)

$a+e+b+c$; Low-mass binaries - Lipunov \& Postnov $(1987,1988)$

$a$; Massive binaries - Dewey \& Cordes (1987)

$a+b+c+d+e$; All masses - Lipunov et al. (1994a,b); see Lipunov (1991)

$a+b+c$; All masses - Tutukov, Yungelson \& Iben (1992)

$a+b+c$; Low-mass binaries - Kolb (1993)

$a+b+c$; Low-mass binaries - Rappaport (1994) (see these proceedings)

$e$; Isolated pulsars - Bailes (1994) (see these proceedings)

$a+b+c$; All masses - Tauris (1994)

\section{Scenario Machine: the Matrix of Galactic Binaries}

In Table 4, we present the main statistical result of the calculation using the Scenario Machine: the distribution of the number of the different types of binaries (Lipunov et al. 1994a,b). We used 500000 artificial binaries.

Here $\alpha_{\mathrm{CE}}, \alpha_{\mathrm{q}}, M_{\mathrm{cr}}$ are the common-envelope parameter, the initial mass ratio exponent, and the minimal presupernova mass which produces a black hole, respectively.

\section{Binary Radio Pulsars with Normal Stars}

After 25 years of radio pulsar observations, a radio pulsar, PSR 1259-63, was discovered in a binary system containing an optical companion; this radio pulsar is paired with a Be-star in a highly eccentric orbit (Johnston et al. 1992). This discovery is an excellent confirmation of the modern theory of evolution of binary stars including the evolution of magnetized compact stars which was basically elaborated before the beginning of the 1980's (Kornilov \& Lipunov 1984).

The natural questions arise - how this eccentricity could be explained in the framework of the theory of binary evolution, and what is the probability to find a radio pulsar on such stretched orbit? To answer these 
TABLE 4. SCENARIO MACHINE: The Matrix of Binary Systems States $\alpha_{\mathrm{CE}}=0.5, \quad \alpha_{\mathrm{q}}=2, \quad M_{\mathrm{cr}}=35 \mathrm{M}_{\odot}, \quad k_{\mathrm{BH}}=0.3, \quad$ kick $=75 \mathrm{~km} \mathrm{~s}^{-1}$

\begin{tabular}{|c|c|c|c|c|c|c|c|c|c|c|c|}
\hline & & \multicolumn{4}{|c|}{ Normal stars } & \multicolumn{3}{|c|}{ White dwarfs } & \multicolumn{2}{|c|}{ Neutr. stars } & \multirow[b]{2}{*}{$\mathrm{BH}$} \\
\hline & & $\mathrm{I}$ & II & III & IV & $\mathrm{E}$ & $\mathrm{P}$ & $\mathrm{A}$ & $\mathrm{E}$ & $\mathrm{P}$ & \\
\hline & I & $\overline{110^{9}}$ & - & - & - & - & - & - & - & - & - \\
\hline & II & $310^{8}$ & $210^{7}$ & - & - & $710^{1}$ & $310^{1}$ & - & - & - & - \\
\hline & III & $110^{2}$ & - & - & - & - & - & - & $410^{-1}$ & $410^{0}$ & - \\
\hline & IIIe & $110^{7}$ & - & $110^{0}$ & - & - & - & - & - & - & - \\
\hline & IV & $210^{4}$ & $410^{2}$ & $210^{-1}$ & $910^{1}$ & $310^{1}$ & $610^{1}$ & - & $310^{0}$ & $210^{-1}$ & - \\
\hline & $\mathrm{E}$ & $210^{5}$ & $110^{5}$ & - & - & $110^{8}$ & $110^{8}$ & $910^{3}$ & $110^{5}$ & $210^{5}$ & - \\
\hline & $\mathrm{P}$ & $210^{2}$ & $210^{4}$ & $910^{4}$ & $310^{2}$ & $110^{8}$ & $110^{8}$ & $310^{4}$ & $610^{4}$ & $110^{5}$ & - \\
\hline $\mathbf{w}$ & $\mathrm{A}$ & $710^{3}$ & $110^{6}$ & $310^{5}$ & $510^{1}$ & $610^{5}$ & $210^{5}$ & $610^{3}$ & - & - & - \\
\hline \multirow[t]{5}{*}{ D } & G & $110^{7}$ & $710^{5}$ & - & $410^{1}$ & $610^{3}$ & $210^{3}$ & - & $110^{5}$ & $210^{5}$ & - \\
\hline & $\mathrm{SA}$ & - & - & $310^{2}$ & - & - & - & - & - & - & - \\
\hline & $\mathrm{M}$ & $510^{6}$ & - & $210^{4}$ & - & - & - & - & $110^{3}$ & - & - \\
\hline & $\mathrm{E}$ & $510^{3}$ & $810^{2}$ & $510^{1}$ & $110^{1}$ & $410^{4}$ & $910^{3}$ & - & $710^{4}$ & $610^{4}$ & $310^{4}$ \\
\hline & $\mathrm{P}$ & $410^{2}$ & $110^{2}$ & $210^{3}$ & $110^{1}$ & $810^{4}$ & $510^{4}$ & - & $310^{4}$ & $210^{5}$ & $610^{4}$ \\
\hline $\mathbf{N}$ & A & $110^{3}$ & $710^{1}$ & $810^{1}$ & $110^{-1}$ & - & - & - & $510^{3}$ & $410^{4}$ & - \\
\hline \multirow[t]{6}{*}{$\mathbf{S}$} & G & $110^{3}$ & $510^{1}$ & - & - & $810^{-1}$ & - & - & - & - & - \\
\hline & $\mathrm{SE}$ & - & - & - & - & - & - & - & - & - & - \\
\hline & SP & - & - & - & - & - & - & - & - & - & - \\
\hline & $\mathrm{SA}$ & - & - & $110^{1}$ & - & - & - & - & - & - & - \\
\hline & $\mathrm{BH}$ & $410^{1}$ & $210^{1}$ & $810^{3}$ & $210^{0}$ & $810^{3}$ & $210^{5}$ & - & $210^{4}$ & $110^{5}$ & $210^{6}$ \\
\hline & SBH & - & - & $810^{1}$ & - & - & - & - & - & - & - \\
\hline
\end{tabular}

questions Lipunov et al. (1994b) have numerically simulated the evolution of an ensemble of binaries with the object of calculating the eccentricity distribution of ejectors in binaries.

We have calculated the distribution of eccentricities of binary radio pulsars with normal stars. The calculation has been done for all pulsars in pairs with normal stars, and separately for visible pulsars (i.e., the optical depth $\tau_{\mathrm{ff}}$ for free-free absorption in the stellar wind is less than 1). The distributions of systems with "visible" pulsars have only one maximum at $e \sim 1$ because the pulsars with high eccentricity are in systems with relatively large semi-major axes (so that $\tau_{\mathrm{ff}}<1$ ). Moreover, these pulsars spend almost all their time at the distant parts of the orbit, which increases the probability of their detection. The probability to find a pulsar in an orbit with the eccentricity $e \geq 0.8$ is at least $\sim 0.90$.

Radio pulsars in binary systems with optical companions were considered in detail by Lipunov \& Prokhorov $(1984,1987)$. The factors of radio wave absorption, of radiation delay due to dispersion measure, and of Fara- 
day rotation in a magnetic field of a stellar wind of an optical companion have been estimated. As a matter of fact, the discovery of such objects gives us a unique possibility for investigating the stellar wind flowing from a massive star, a sort of radio probe launched by nature itself. All three effects have recently been discovered (see Johnston 1994).

\section{Binary Radio Pulsars with Black Holes}

The discovery of binary radio pulsars with massive unseen companions ( $>3$ to $5 \mathrm{M}_{\odot}$ ) would be of great importance for fundamental physics and the modern theory of stellar evolution, giving compelling evidence for blackhole existence in nature. The formation of binaries consisting of a black hole (BH) and a radio pulsar (PSR) has been previously discussed by BisnovatyiKogan \& Komberg (1974) and Narayan et al. (1991). As is well known, observations of a radio pulsar in a binary system (Manchester \& Taylor 1977; Brumberg et al. 1975; Blandford \& Teukolsky 1975) provide the most accurate information about physical parameters of the binary companion. This concerns not only the mass of the companion, which until now has been the main signature of $\mathrm{BH}$. The pulsar radio emission can be used as a probe of plasma emitted by the secondary star (Lipunov \& Prokhorov 1984; Lipunov et al. 1994b) and, consequently, by giving a picture of the physical properties of the adjacent medium, can prove the $\mathrm{BH}$ nature of the companion. Some relativistic effects specific to $\mathrm{BH}$ can be observed in these systems, such as propagation of the radiation through the $\mathrm{BH}$ ergosphere.

The calculations with the Scenario Machine predict a subclass of binary radio pulsars with black holes to exist, their galactic number being large enough (Lipunov et al. 1994a) for them to be discovered in the near future. The pulsars themselves must be similar to standard isolated pulsars.

Recently, the 1-s PSR B0042-73 in a highly eccentric $(e=0.8)$ 51-day orbit around a massive companion was discovered in the Small Magellanic Cloud (Kaspi et al. 1994). This may be the first such pulsar with a BH (see, however, the contribution by Kaspi to these proceedings). The fact that the first pulsar discovered in the SMC proved to be a binary seems natural in the framework of a recent burst of star formation in the SMC. Lipunov et al. (1994c) confirm this fact by a numerical calculation of the evolution of radio pulsars after a star formation burst.

\section{References}

Bailes, M. 1994, these Proceedings

Bisnovatyi-Kogan, G.S. \& Komberg, B.V. 1974, AZh 51, 373

Bisnovatyi-Kogan, G.S. \& Komberg, B.V. 1975, AZh 52, 457

Blandford, R.D. \& Teukolsky, S.A. 1975, ApJ 198, L27

Brumberg, V.A. et al. 1975, SvAL 1, 5 
Davies, R.E. \& Pringle, J.E. 1981, MNRAS 196, 209

Dewey, R.J. \& Cordes, J.M. 1987, ApJ 321, 780

Johnston, S. et al. 1992, ApJ 387, L37

Johnston, S. 1994, these Proceedings

Mlarionov, A.F. \& Sunyaev, R.A. 1975, A\&A 39, 185

Kaspi, V.M. et al. 1994, ApJ 423, L43.

Kolb, U. 1993, in Proceedings International Workshop in Memory of Livio Gratton (Monte Porzio, 21 June 1993), (in press)

Kornilov, V.G. \& Lipunov, V.M. 1983a, AZh 60, 284

Kornilov, V.G. \& Lipunov, V.M. 1983b, AZh 60, 574

Kornilov, V.G. \& Lipunov, V.M. 1984, AZh 61, 686

Lipunov, V.M. 1982a, Ap\&SS 85, 451

Lipunov, V.M. 1982b, Pis'ma AZh 8, 358

Lipunov, V.M. 1984, Adv. Space Res. 3, 323

Lipunov, V.M. 1987, Ap\&SS 132, 1

Lipunov, V.M. 1991, in Frontier Objects in Astrophysics and Particle Physics, Vulcano Workshop 1990, F. Giovannelli and G. Mannocchi (Eds.), Italian Physical Society, p. 29

Lipunov, V.M. 1992, Astrophysics of Neutron Stars, Springer-Verlag

Lipunov, V.M. \& Postnov, K.A. 1987, Astr. Zh. 64, 773

Lipunov, V.M. \& Postnov, K.A. 1988, Astr. Sp. Sc. 145, 1

Lipunov, V.M. \& Prokhorov, M.E. 1984, Ap\&SS 98, 221

Lipunov, V.M. \& Prokhorov, M.E. 1987, AZh 64, 1189

Lipunov, V.M. \& Shakura, N.I. 1976, Pis'ma AZh 2, 343

Lipunov, V.M. et al. 1994a, ApJ 423, L121

Lipunov, V.M. et al. 1994b, A\&A 282, 61

Lipunov, V.M., Postnov, K.A. \& Prokhorov, M.E. 1994c, ApJ (in press)

Lipunov, V.M., Postnov, K.A. \& Prokhorov, M.E. 1995, A\&A (in press)

Manchester, R.N. \& Taylor, J.H. 1977, Pulsars, Freeman

Narayan, R., Piran, T. \& Shemi, A. 1991, ApJ 379, L17

Osminkin, E.Yu. \& Prokhorov, M.E. 1995, SvAL (in press)

Paczyński, B. 1971, ARA\&A, 9, 183

Rappaport, S. 1994, these Proceedings

Savonije, G.J. \& Van den Heuvel, E.P.J. 1977, ApJ 214, L19

Shakura, N.I. 1975, Pis'ma AZh 1, 23

Shvartsman, V.F. 1970, Radiofizika 13, 1852

Shvartsman, V.F. 1971, AZh 48, 438

Tatarintzeva, V.S. et al. 1989, in Proc. 23rd ESLAB Symposium on Two Topics in X-ray Astronomy, ESA SP-296, p. 653

Tauris, T.M. 1994, in XXIInd IAU General Assembly Posters, H. van Woerden (Ed.), p. 110

Tutukov, A.V. \& Yungelson, L.R. 1973, Astrofizika 8, 381

Tutukov, A.V., Yungelson, L.R., Iben Jr, I. 1992, ApJ 386, 197

Van Kerkwijk, M.H. et al. 1992, Nat 355, 703

Van den Heurel, E.P.J. 1977, Ann. N.Y. Acad. Sci. 302, 13

Van den Heuvel, E.P.J. \& Heise, J. 1972, Nat. Phys. Sci. 239, 67

Wickramasinghe, D.T. \& Whelan, J.A.J. 1975, Nat 258, 502 